\title{
Dimethyl Sulfoxide-Free and Water-Soluble Fluorescent Probe for Detection of Bovine Serum Albumin Prepared by Ionic Co- assembly of Amphiphiles
}

Qin Tong, Weichun Wu, Jianghong Hu, Junhao Wang, Ke Li, Bin Dong, Bo Song*

College of Chemistry, Chemical Engineering and Materials Science, Soochow University, Suzhou 215123, P. R. China. E-mail: songbo@suda.edu.cn

\section{Table of Contents:}

Figure S1. UV-vis and fluorescence spectra of DPP-12 in aqueous solution S3

Figure S2. Plot of fluorescence intensity at $595 \mathrm{~nm}$ versus the corresponding concentration of DPP-12.

Figure S3. Photographs of the solutions of DPP-12 in presence of BSA and SDBS.

Figure S4. Fluorescence spectra of DPP-12 in presence of different concentrations of (a) ST and (b) SL. ..S4 
Figure S5. (a) Fluorescence spectra of mixed solution of DPP-12 and SDBS in presence of different concentrations of BSA. (b) Plot of fluorescence intensity at $595 \mathrm{~nm}$ versus concentration of BSA. S5

Figure S6. Fluorescence spectra of SDBS in presence of different concentrations of BSA.

Figure S7. (a) Fluorescence spectra of unknown samples from newborn calf serum after diluting. (b) UV-vis absorption of newborn calf serum being diluted to different concentrations. .S6

Figure S8. Concentration dependent absorbance of BSA at $280 \mathrm{~nm}$, and linear fitting of the data. ..S6

Figure S9. ${ }^{1} \mathrm{H} \mathrm{NMR}$ spectrum of compound $\boldsymbol{b}$ in $\mathrm{CDCl}_{3}$. S7

Figure S10. ${ }^{1} \mathrm{H}$ NMR spectrum of compound $c$ in $\mathrm{CDCl}_{3}$. S7

Figure S11. ${ }^{1} \mathrm{H}$ NMR spectrum of compound $\boldsymbol{d}$ in $\mathrm{CDCl}_{3}$ S8

Figure S12. ${ }^{1} \mathrm{H}$ NMR spectrum of DPP-12 in DMSO- $d_{6}$ S8

Figure S13. ${ }^{13} \mathrm{C}$ NMR spectrum of DPP-12 in DMSO- $d_{6}$ S9

Figure S14. MALDI-TOF MS of compound $\boldsymbol{b}$. S9

Figure S15. ESI-MS of compound $c$. $\mathrm{S} 10$

Figure S16. MALDI-TOF MS of compound $\boldsymbol{d}$. $\mathrm{S} 10$

Figure S17. MALDI-TOF MS of DPP-12. S1 1 


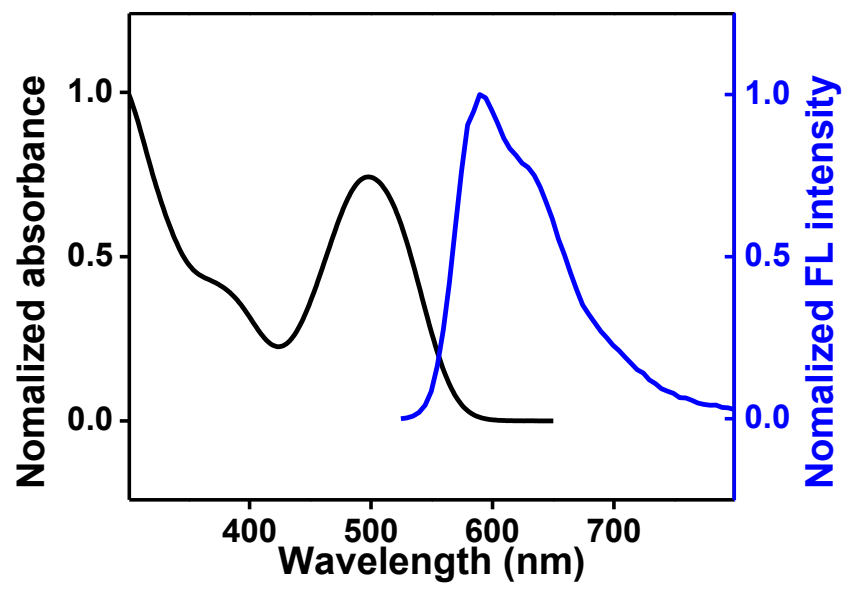

Figure S1. UV-vis and fluorescence spectra of DPP-12 in aqueous solution. The concentration of DPP-12 is $1 \times 10^{-4} \mathrm{~mol} \mathrm{~L}^{-1} \cdot \lambda_{\mathrm{ex}}=505 \mathrm{~nm}$.

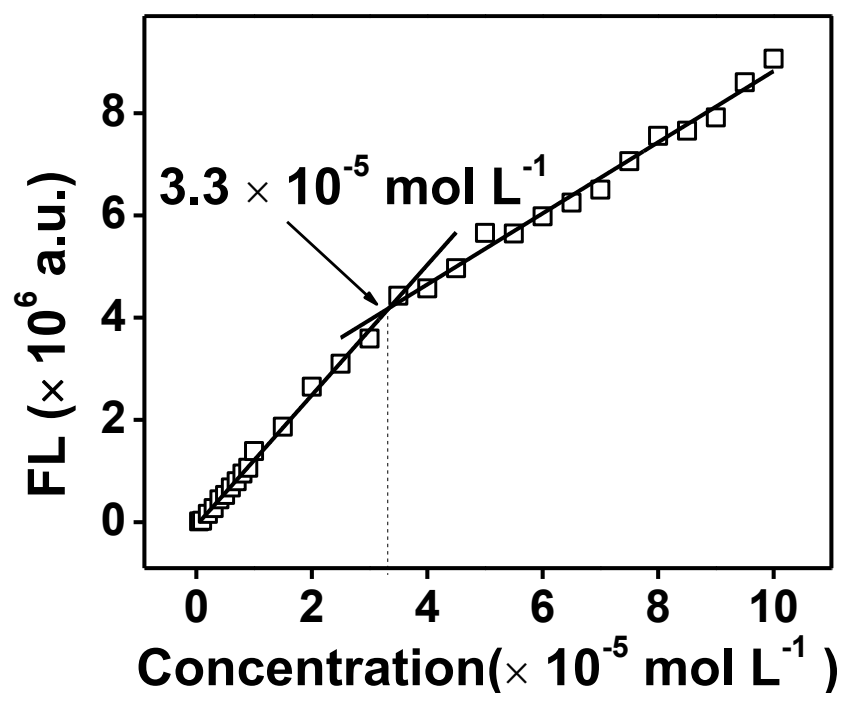

Figure S2. Plot of fluorescence intensity at $595 \mathrm{~nm}$ versus the corresponding concentration of DPP-12. $\lambda_{\mathrm{ex}}=505 \mathrm{~nm}$.

DPP-12 forms a uniform aqueous solution. Addition of BSA resulted in precipitation. No matter how much SDBS was added in the DPP-12 solution, there is no precipitate was observed. 


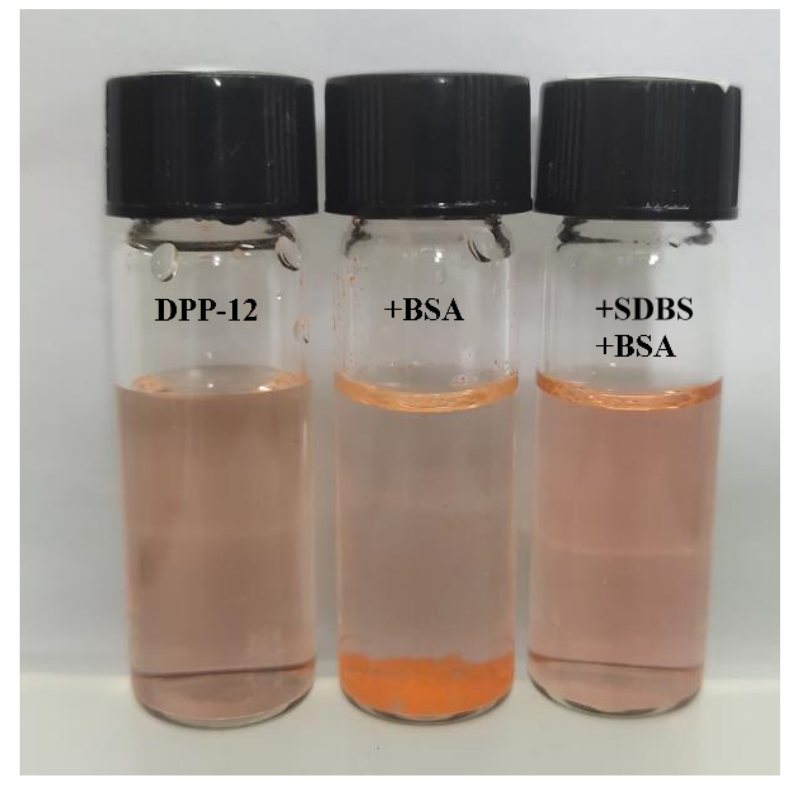

Figure S3. Photographs of the solutions of DPP-12 in presence of BSA and SDBS.
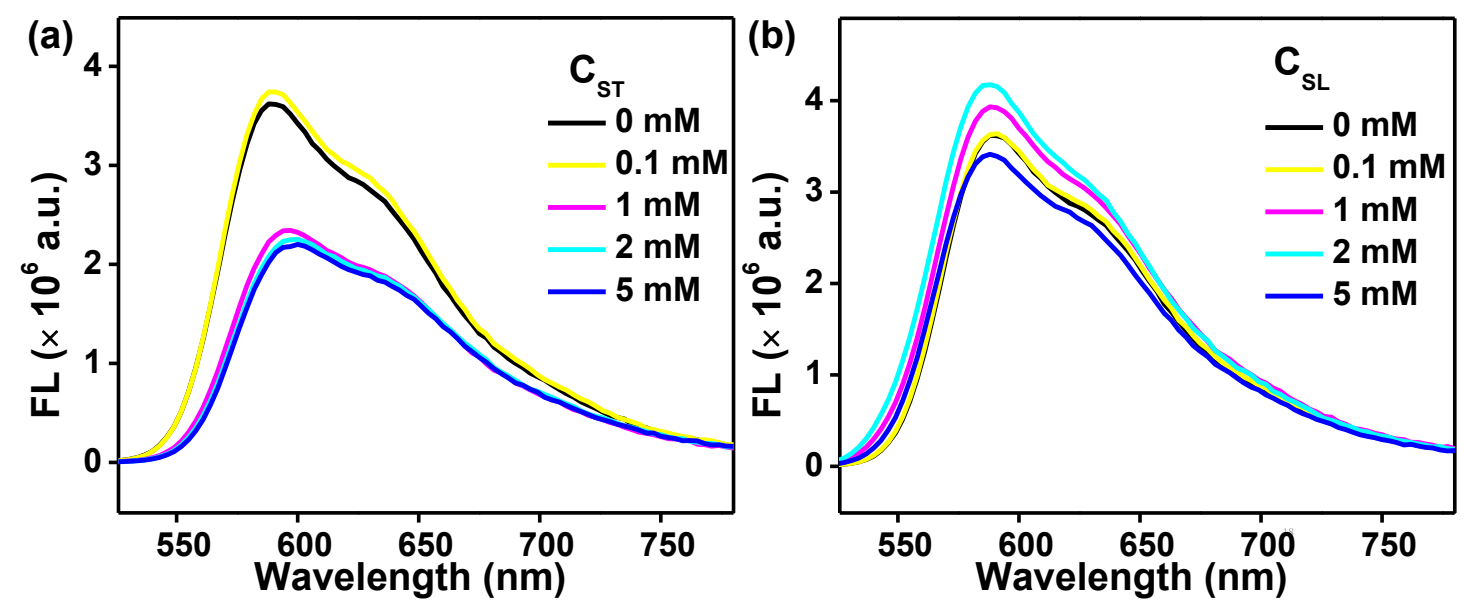

Figure S4. Fluorescence spectra of DPP-12 in presence of different concentrations of (a) ST and (b) SL. 

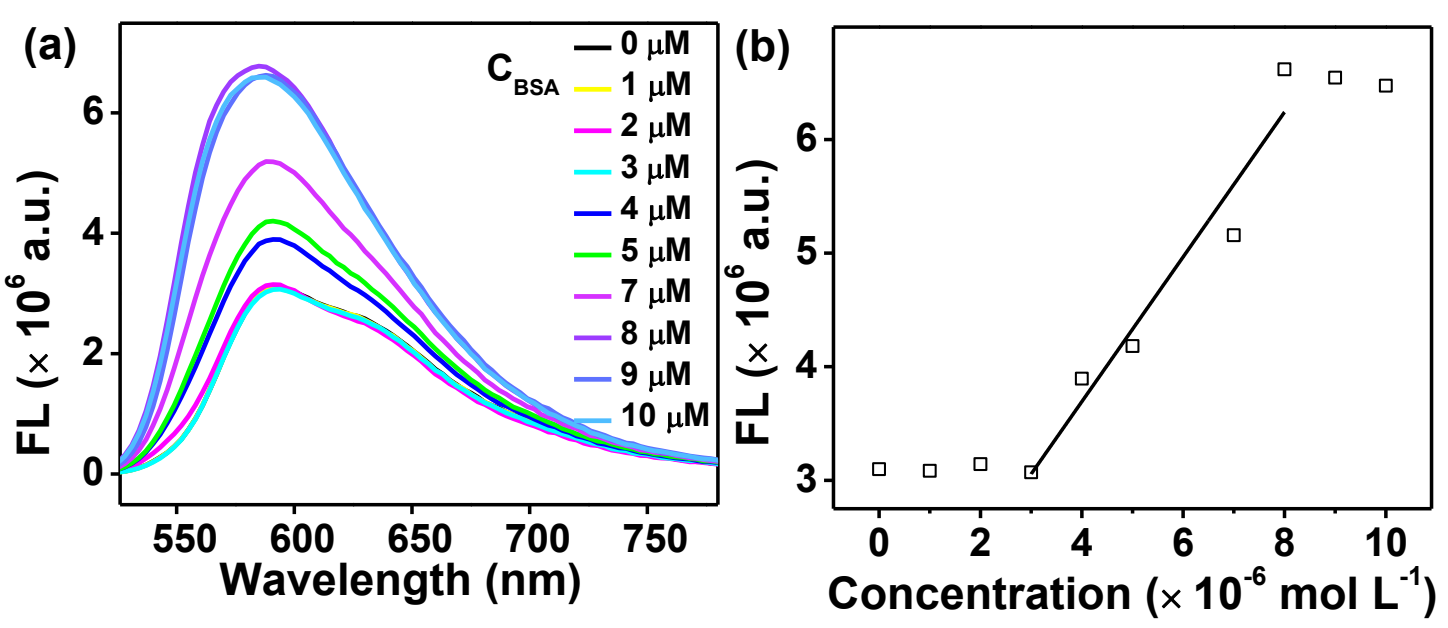

Figure S5. (a) Fluorescence spectra of mixed solution of DPP-12 and SDBS in presence of different concentrations of BSA. (b) Plot of fluorescence intensity at $595 \mathrm{~nm}$ versus concentration of BSA. $C_{\mathrm{SDBS}}=2 \times 10^{-3} \mathrm{~mol} \mathrm{~L}^{-1}$.

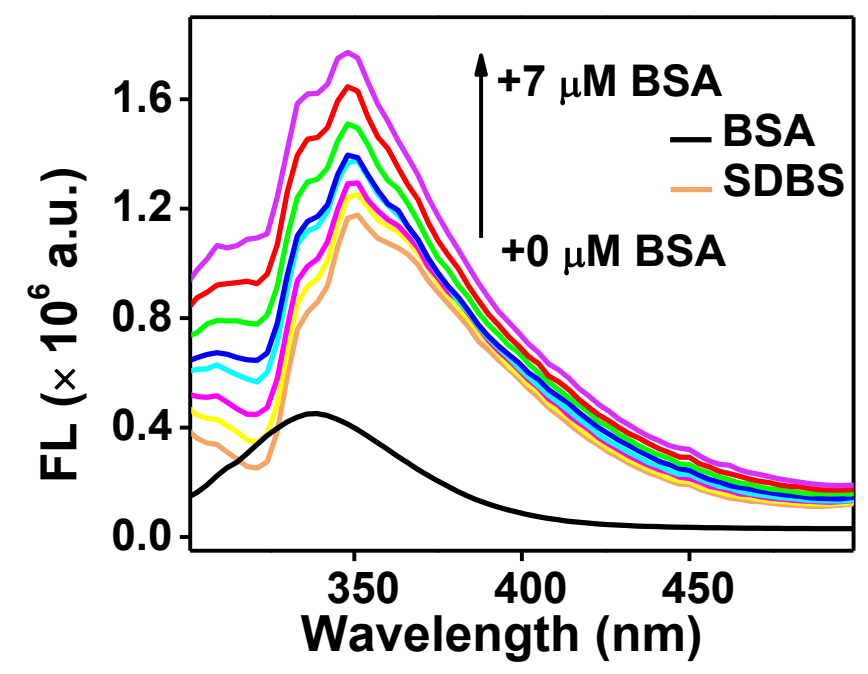

Figure S6. Fluorescence spectra of SDBS in presence of different concentrations of BSA. 

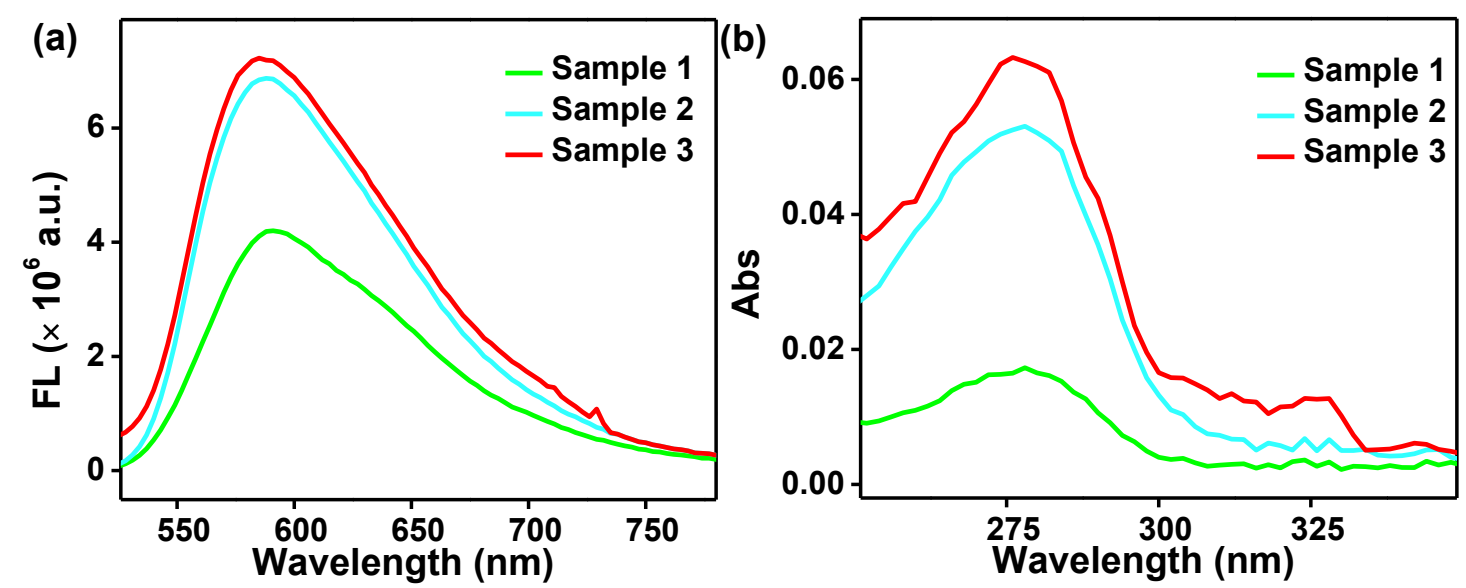

Figure S7. (a) Fluorescence spectra of unknown samples from newborn calf serum after diluting. (b) UV-vis absorption of newborn calf serum being diluted to different concentrations.

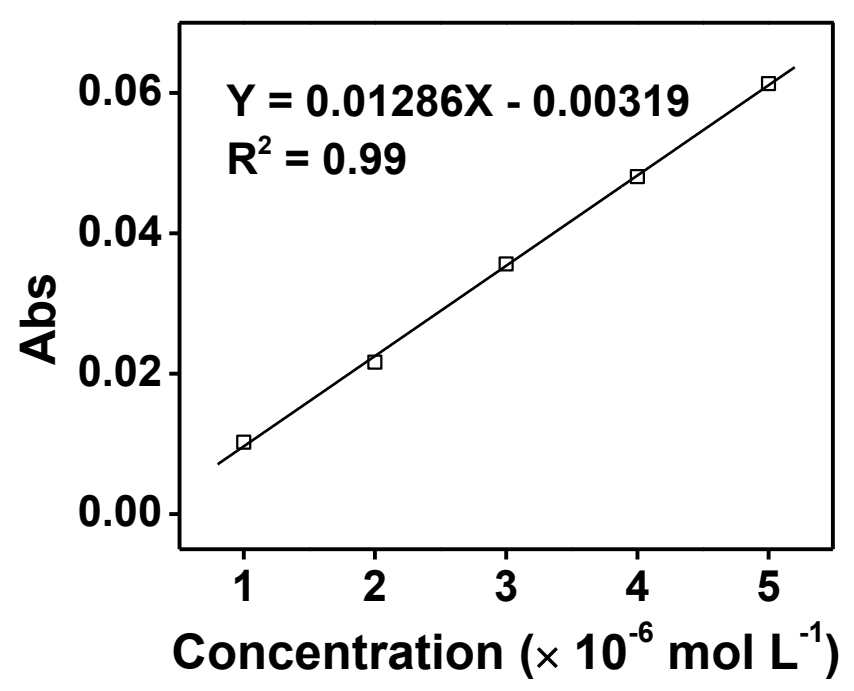

Figure S8. Concentration dependent absorbance of BSA at $280 \mathrm{~nm}$, and linear fitting of the data. 


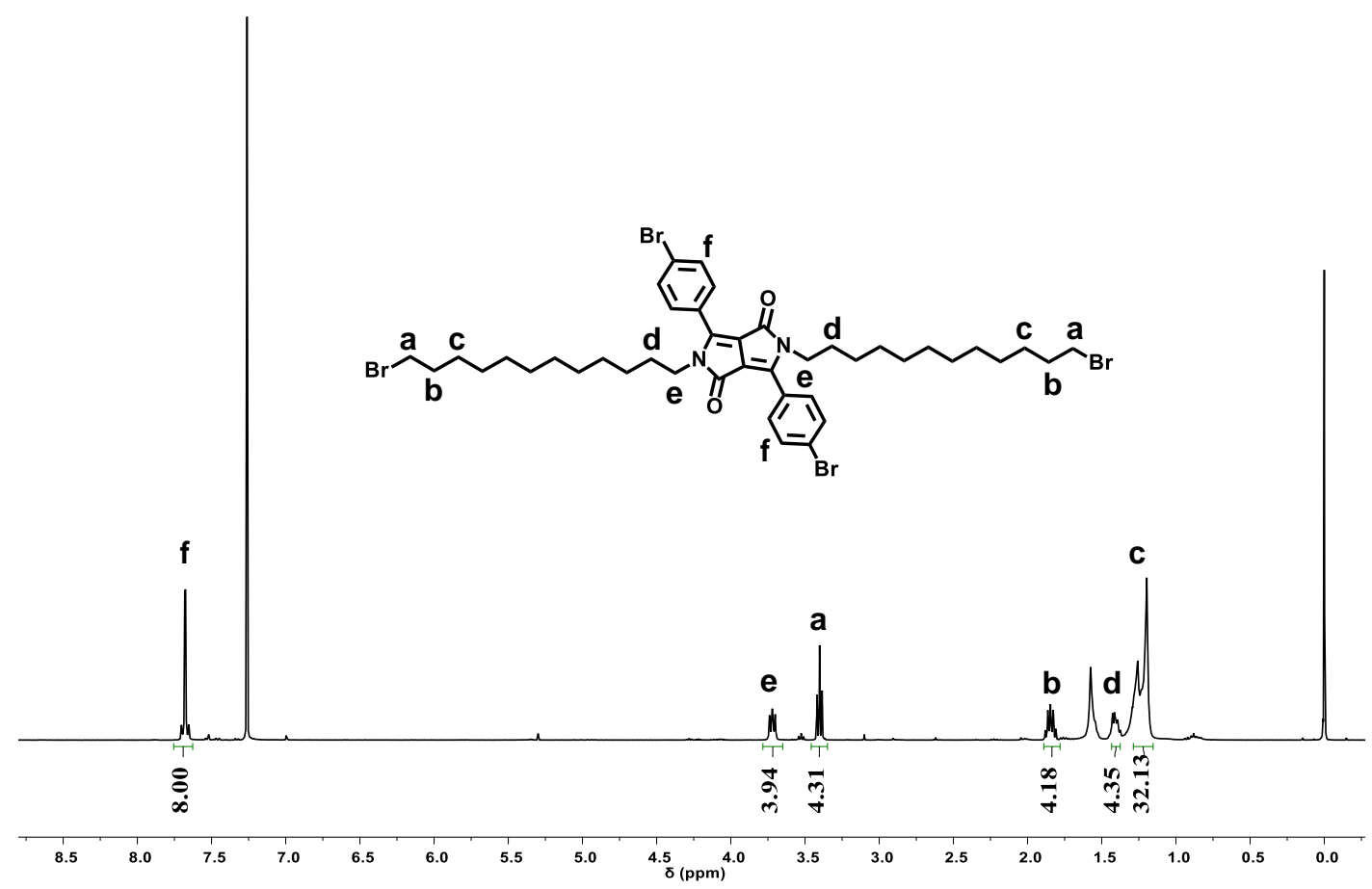

Figure S9. ${ }^{1} \mathrm{H}$ NMR spectrum of compound $\boldsymbol{b}$ in $\mathrm{CDCl}_{3}$.

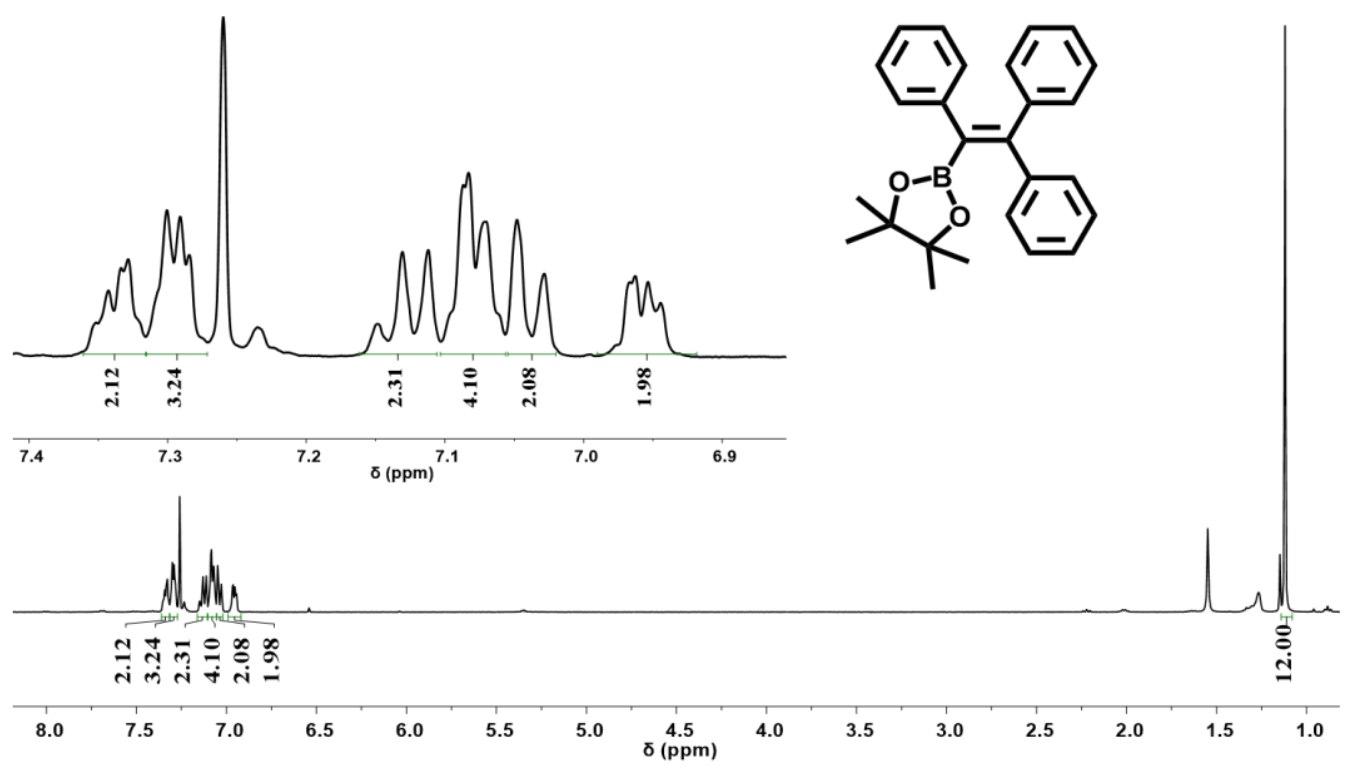

Figure S10. ${ }^{1} \mathrm{H}$ NMR spectrum of compound $c$ in $\mathrm{CDCl}_{3}$. 

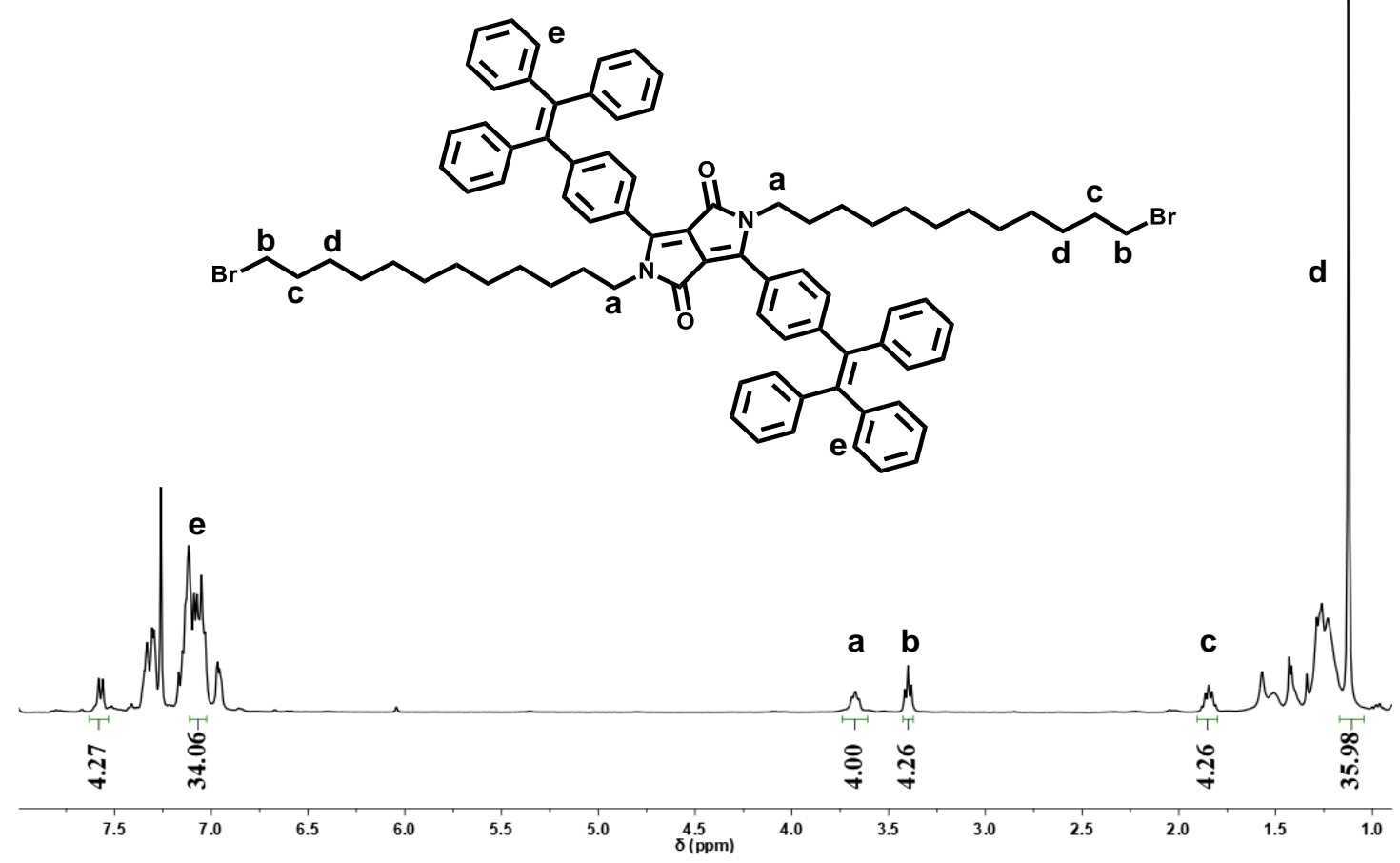

Figure S11. ${ }^{1} \mathrm{H}$ NMR spectrum of compound $\boldsymbol{d}$ in $\mathrm{CDCl}_{3}$.

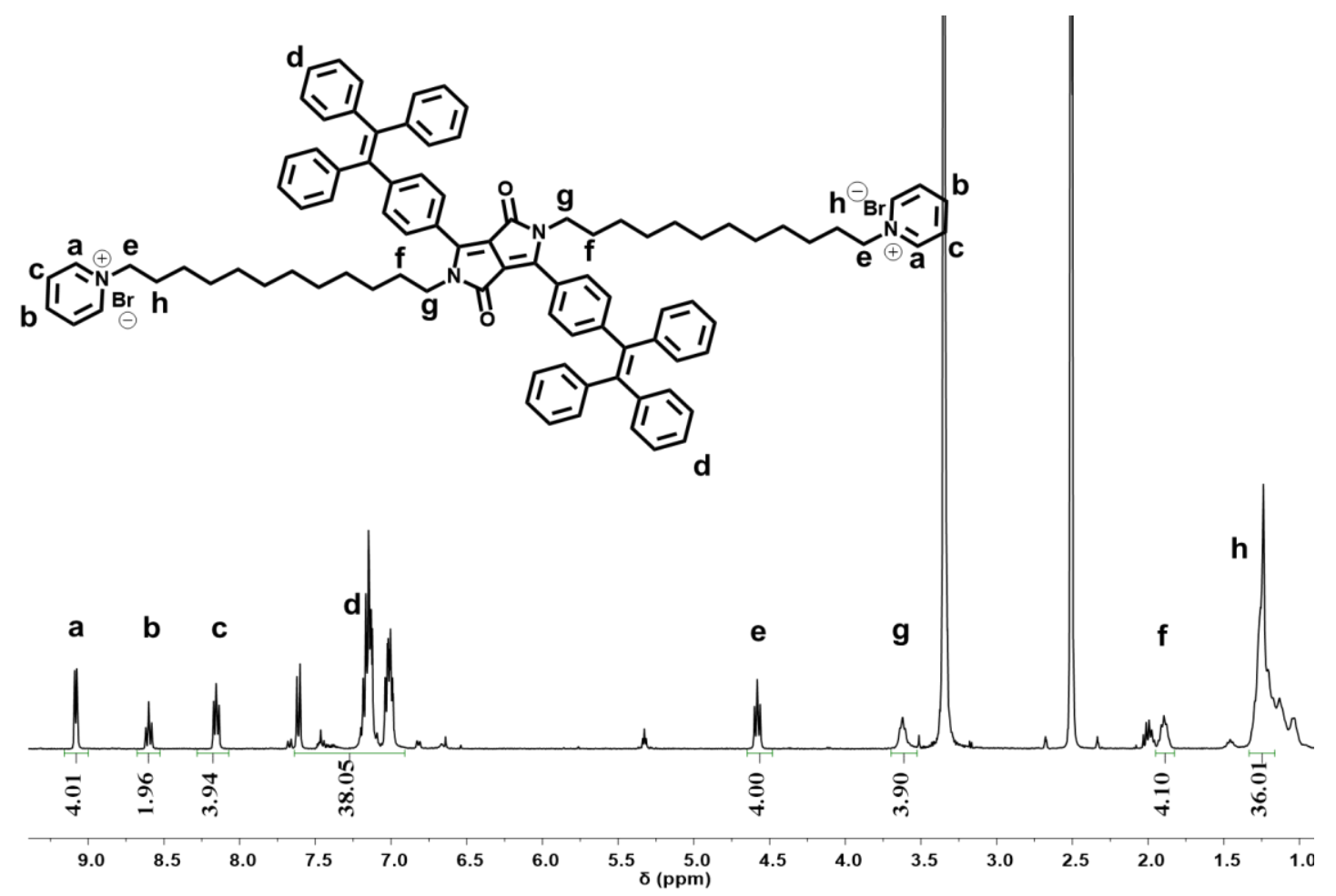

Figure S12. ${ }^{1} \mathrm{H}$ NMR spectrum of DPP-12 in DMSO- $d_{6}$. 


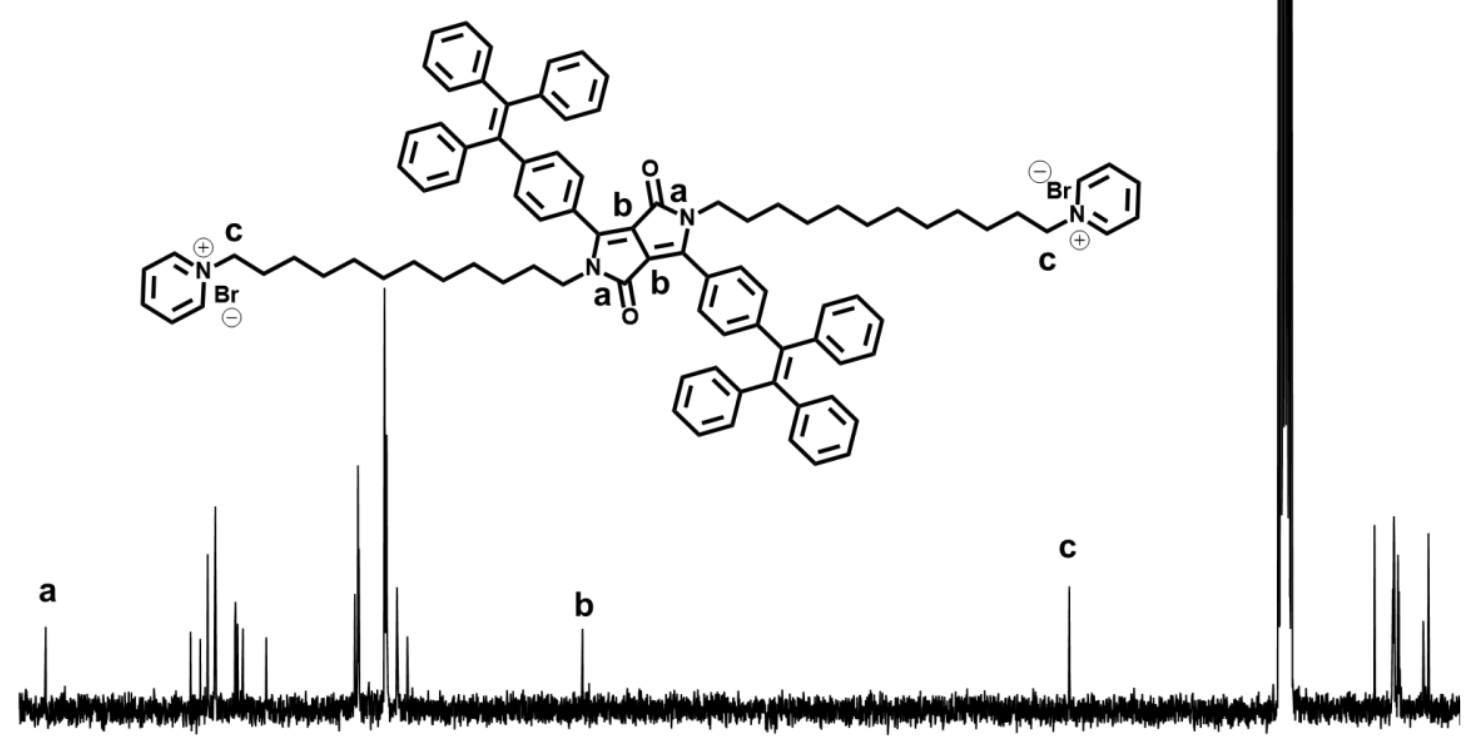

$\begin{array}{llllllllllllllllllllllllllll}160 & 155 & 150 & 145 & 140 & 135 & 130 & 125 & 120 & 115 & 110 & 105 & 100 & \begin{array}{c}95 \\ \delta(\mathrm{ppm})\end{array} & 85 & 80 & 75 & 70 & 65 & 60 & 55 & 50 & 45 & 40 & 35 & 30 & 25\end{array}$

Figure S13. ${ }^{13} \mathrm{C}$ NMR spectrum of DPP-12 in DMSO- $d_{6}$.

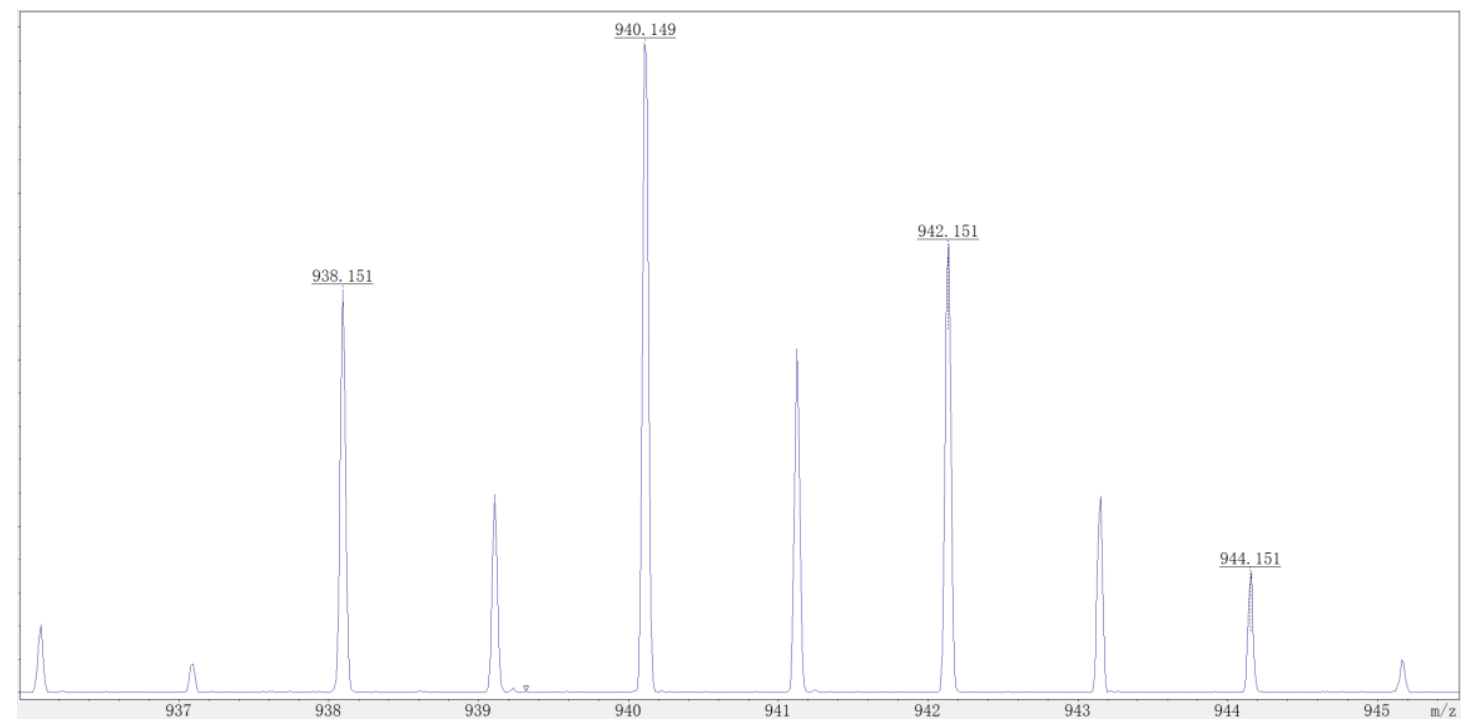

Figure S14. MALDI-TOF MS of compound $\boldsymbol{b}$. 


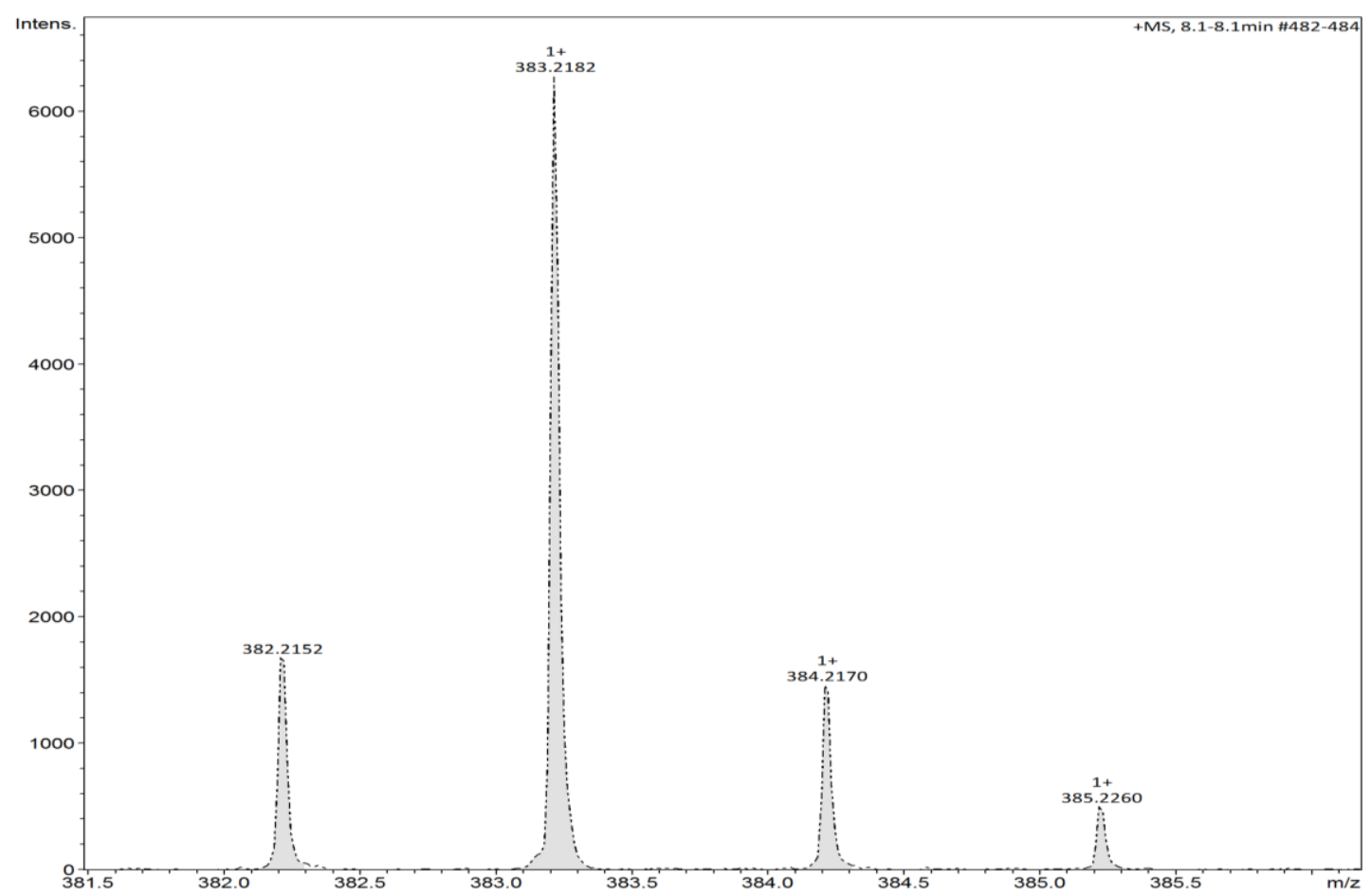

Figure S15. ESI-MS of compound $c$.

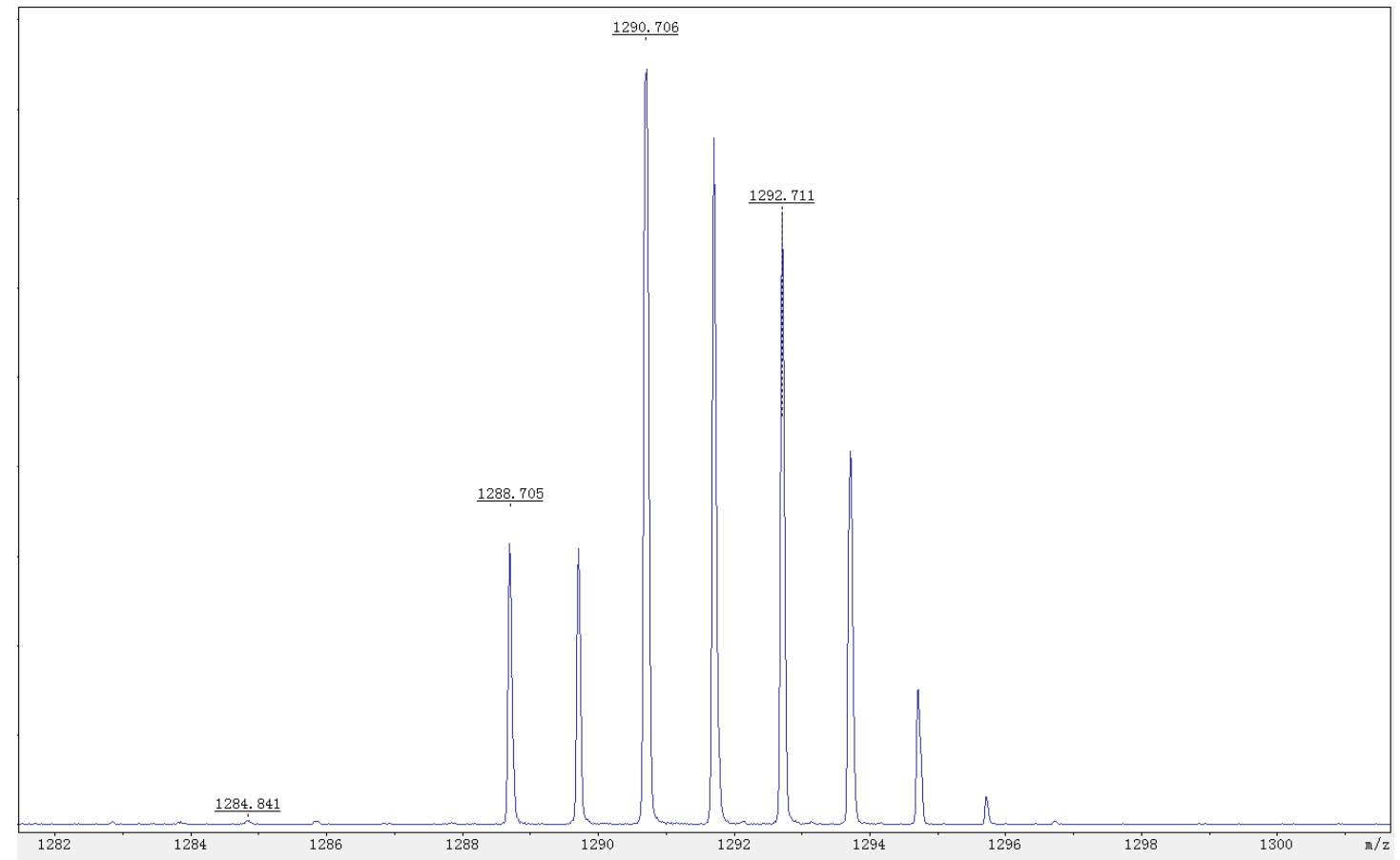

Figure S16. MALDI-TOF MS of compound $\boldsymbol{d}$. 


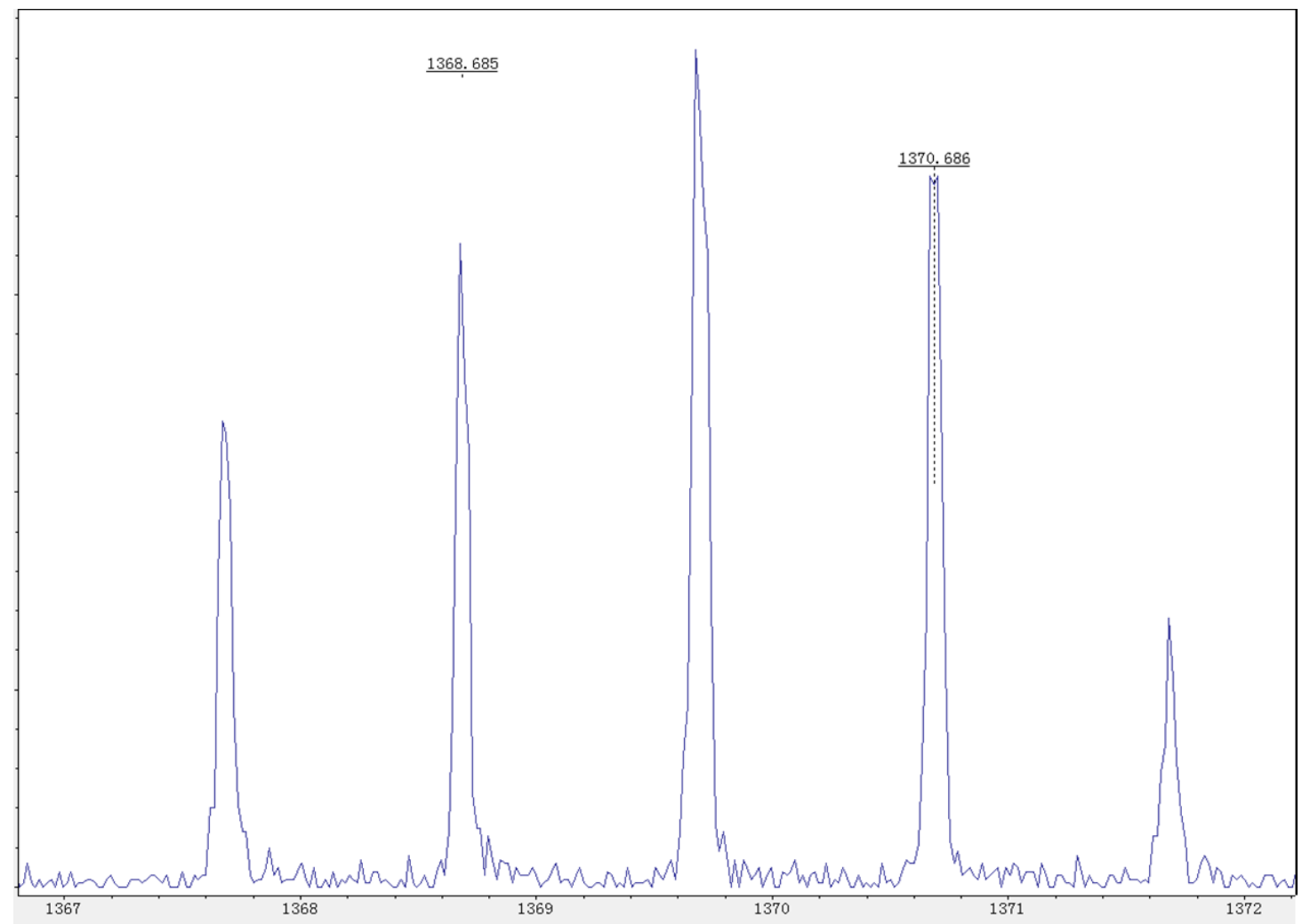

Figure S17. MALDI-TOF MS of DPP-12. 